\title{
Redundancy and embedded exhaustification *
}

\author{
Marie-Christine Meyer \\ The Hebrew University of Jerusalem
}

\begin{abstract}
I show how a formalization of Grice's Brevity intuition, which I call Efficiency (Meyer 2013, 2014), correctly distinguishes between acceptable and unacceptable disjunctions that all seem to be redundant at first (e.g., Gajewski \& Sharvit 2012; Mayr \& Romoli 2013). The upshot is that the presence of embedded implicatures is one way of making a structure efficient in the formal sense developed here. I show that a particular prediction of Efficiency-the existence of embedded implicatures resulting in overall weakening of meaning - is not incorrect, and sheds new light on the role of rise-fall-rise intonation in these cases (Fox \& Spector 2013; Büring 1997).
\end{abstract}

Keywords: Implicatures, Disjunction, Economy, Redundancy, Exhaustification, Embedded implicatures, Contrastive-Topic Intonation, Meta-linguistic negation

\section{Introduction}

\subsection{Background}

The nature of scalar implicatures remains a debated topic. Some theories view the step from some to not all as an instance of rational inferences not specific to language. Others propose that the inference arises instead through a languagespecific mechanism (e.g., Sauerland 2011 and references therein). Specifically, proponents of the grammatical theory of scalar implicatures assume a covert operator that strengthens the semantic denotation of its (propositional) argument by adding the negation of certain scalar alternatives. For example:

$$
\llbracket \operatorname{exh}[\mathrm{A} \text { or } \mathrm{B}] \rrbracket=(A \vee B) \wedge \neg(A \wedge B)
$$

If we accept that exh is available in the functional lexicon, the question arises as to what principles guide its syntactic distribution. In this paper I suggest that this is partly predicted by a constraint against redundancy, which may itself be a language-

* I thank Sigrid Beck, Luka Crnič, Danny Fox, Yosef Grodzinsky, Irene Heim, and audiences at SALT 25, MIT LingLunch, and the Tel Aviv Linguistics Colloquium for valuable questions and comments. Thanks also to the SALT 25 reviewers for helpful comments. 
independent, rational principle as envisaged by Grice. The main ideas to be defended are thus the following:

- The distribution of exh is restricted by a pragmatic principle of Efficiency

- Efficiency rules out redundant structures if there is a simpler competitor

- Insertion of exh can sometimes rescue an otherwise redundant structure

\subsection{Data}

Consider the following two formulae:
a. $A \vee B$
b. $\quad A \vee(\neg A \wedge B)$

The two are equivalent. ${ }^{1}$ But (2b) is a redundant formula given the availability of the shorter (2a). This might lead to expectations about the corresponding natural language structures, especially if we follow Grice in assuming that language use is guided by a principle constraining prolixity: We might expect a contrast in acceptability:

(3) a. Mary studied math or she studied physics.

b. Mary studied math or she didn't and she studied physics.

Contrary to our expectations, both the prolix and the briefer, more efficient version are licensed. In view of this, consider now a second minimal pair:

a. Mary studied math or physics or both.

b. \# Mary didn't study math or physics or both.

Both sentences seem to be redundant in the same way that ( $3 b)$ is; compare the corresponding logical formulae:

$$
\begin{array}{ll}
\text { a. } & (A \vee B) \vee(A \wedge B) \equiv(A \vee B) \\
\text { b. } & \neg[(A \vee B) \vee(A \wedge B)] \equiv \neg(A \vee B)
\end{array}
$$

1 The proof is trivial:

$$
\text { (i) } \quad \begin{aligned}
A \vee(\neg A \wedge B) \\
\equiv(A \vee \neg A) \wedge(A \vee B) \\
\equiv \top \wedge(A \vee B) \\
\equiv(A \vee B)
\end{aligned}
$$


Redundancy and embedded exhaustification

But whereas (4a) is felicitous, the equally redundant (4b) sounds odd. The questions raised by the two pairs in (3) and (4) are thus:

\section{Q: What distinguishes felicitous from infelicitous redundancy?}

Q-1: Why is a seemingly redundant sentence of the form $A$ or not $A$ and $B$ licensed?

Q-2: Why is a seemingly redundant sentence of the form $A$ or $B$ or both licensed, when its equally redundant negation not [A or $B$ or both] is not?

It is beyond the scope of this paper to develop a general theory of redundancy; my goal here is to contribute to this broader agenda by addressing the two specific sub-questions stated above, thus extending some previous work in this area (Meyer 2013, 2014). To anticipate, I will argue that felicitous cases of (seeming) redundancy involve LFs that are made non-redundant by insertion of exh. The infelicitous cases are such that no adjunction site of exh would yield a non-redundant LF. This answer entails that the simple logical formulae above do not in fact capture the semantics of the corresponding sentences-it's more complicated.

\section{Core proposal}

Above I alluded to the Gricean intuition that language use obeys a principle of brevity. In previous work I have proposed the following formalization of this intuition:

\section{Efficiency}

An LF $\phi$ is ruled out if there is a distinct competitor $\psi$ such that (i) $\psi<\phi$ and (ii) $\llbracket \psi \rrbracket \equiv \llbracket \phi \rrbracket$

The idea is to use Katzir (2007)'s notion of structural complexity to define a set of competitors against which redundancy of a given LF is evaluated. Structural complexity is defined in terms of the 'at-most-as-complex' relation $\lesssim . \psi \lesssim \phi$ iff $\psi$ can be derived from $\phi$ by substituting terminal nodes in $\phi$ with items from the lexicon (e.g., replace or with and), and/or substituting $\phi$ with a subconstituent (e.g., substitute $A$ for $A$ or $B$; see Katzir (2007); Fox \& Katzir (2011); Trinh \& Haida (2015) for details). $\psi$ is strictly simpler than $\phi, \psi<\phi$, iff $\psi \lesssim \phi \wedge \neg(\phi \lesssim \psi)$.

Going back to the pair in (3), we can thus ask why $A$ or not $A$ and $B$ is acceptable, even though there seems to be a strictly simpler, equivalent competitor $A$ or $B$. This is the first sub-question from above (Q-1), and we will address it in what follows. 
Addressing Q-1 First consider the simplest LF for the complex disjunction:

\section{LF1}

Mary studied math or [she didn't study math and she studied physics]

The competitors for such an LF include the simple disjunction: ${ }^{2}$

$$
\mathcal{C O} \mathcal{M P}(\mathrm{LF} 1)=\{\text { Mary studied math or she studied physics, } \ldots\}
$$

It is easy to see that this competitor is semantically equivalent, and strictly simpler than LF1. LF1 is thus ruled out by Efficiency.

I assume a covert exhaustivity operator exh with the following semantics roughly, it adds to its argument $\phi$ the negation of $\phi$ 's alternatives in keeping with consistency (I use sloppy notation in not distinguishing between object- and metalanguage):

$$
\begin{aligned}
& \llbracket \operatorname{exh} \phi \rrbracket=\phi \wedge \forall \psi \in I E(\phi): \neg \psi \\
& \psi \in I E(\phi) \text { iff } \psi \in \mathcal{A L T}(\phi) \text { and } \neg(\phi \rightarrow \psi) \text { and there is no } \chi \text { s.t. } \chi \in \mathcal{A L T}(\phi) \\
& \text { and } \phi \wedge \neg \psi \rightarrow \chi^{3}
\end{aligned}
$$

Consider the definition of the set $I E$ of innocently excludable alternatives: even though $A \in \mathcal{A} L T(A$ or $B), A$ is not in the set $I E(A$ or $B)$ because $\neg A \wedge(A \vee B) \rightarrow B$, and $B$ is itself in $\mathcal{A} L T(\phi)$, since $B \lesssim[A$ or $B]$. Together, then, the standard scalar implicature of disjunction will be derived with the following LF (using transparent abbreviations):

\section{LF2}

exh [Mary studied math or [she didn't study math and she studied physics]] $I E=\{\perp,[\text { math and physics }]\}^{4}$

$=$ Mary studied math or physics \& $\neg($ Mary studied math and physics)

2 Recall that competitors are those structures that are strictly simpler than LF1, in the technical sense defined below (6). Roughly, competing structures are those that can be derived by substitution of terminal nodes and substitution with subconstituents in LF1. The particular competitor given below can be derived in two ways. Following Katzir 2007, we may allow for a deletion operation: [Mary studied math or she didn't study math and she studied physics]. Here I followed Fox \& Katzir (2011); Trinh \& Haida (2015) in only allowing for substitution with sub-constituents. To derive the above competitor by substitution, the sub-constituent [she didn't study math and she studied physics] can be substituted by its underlined subconstituent within the full structure.

3 This is a simplified semantics which, for the examples discussed in this paper, is fully sufficient. For a more fine-grained semantics see Fox (2007).

4 Using Katzir's algorithm, the set of structures $\psi$ s.t. $\psi \lesssim$ LF2 is the following: $\mathcal{A} L T$ (math or [ $\neg$ math and physics $])=\{$ math, $[\neg$ math and physics $]$, math and $[\neg$ math and physics $](=\perp), \ldots .$.$\} . Since the$ argument of exh, [math or [ $\neg$ math and physics]] is equivalent to a simple disjunction math or physics, the only alternatives that exh can negate is the one given above. 
Redundancy and embedded exhaustification

Given the principle of Efficiency, this LF will only be licensed if there is no simpler competitor that expresses the same exclusive disjunctive meaning. Such a competitor does exist, however, ruling out LF2 as well:

$\mathcal{C O M P}(\mathrm{LF} 2)=\{$ exh $[$ Mary studied math or she studied physics $], \ldots\}$ $=$ Mary studied math or physics \& $\neg$ (Mary studied math and physics)

Consider now the effect of embedded exhaustification as in LF3:

\section{LF3}

[exh Mary studied math] or [she didn't study math and she studied physics]

I assume that the only relevant alternative for the first disjunct is given by she studied physics. ${ }^{5}$

$\llbracket \mathrm{LF} 3 \rrbracket=($ Mary studied math and not physics $)$ or she studied physics and not math

This is an exclusive disjunctive meaning. Schematically:

$$
\begin{aligned}
& \text { LF3 } \\
& [[\operatorname{exh} \mathrm{A}] \text { or [not A and } \mathrm{B}]] \\
& =A \nabla B
\end{aligned}
$$

Is LF3 efficient? An exclusive disjunction is standardly expressed by a structure of the form exh [A or B], which seems to be structurally simpler than LF3. Recall that LF2 was also blocked by such a simple LF. Crucially, however, LF3 is not in competition with such a structure. This is because Efficiency is defined in terms of the structure-sensitive relation $\lesssim:$ An LF $\phi$ will only be blocked by competitors derivable from $\phi$ via a restricted set of syntactic operations-intuitively, those operations which do not increase complexity. As it turns out, there is no way of generating a simpler, equivalent competitor for LF3 which satisfies these conditions. In particular, the standard LF for exclusive disjunctions is not in fact in structural competition with LF3:

5 There can be many other structural alternatives, e.g., Mary got drunk. These will not have any effect on the result. Assume that the embedded exh in LF3 negates further alternatives. I assume that alternatives have to stay constant across competitors when checking Efficiency. The fact remains that there will be no simpler competitor to express this meaning and that in particular, LF* is not a structural competitor. In view of additional alternatives, if it were a competitor, it would no longer be equivalent. 
(16)

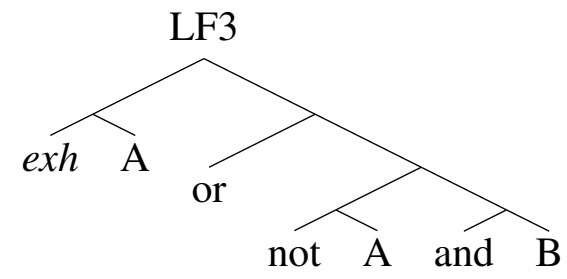

(17)

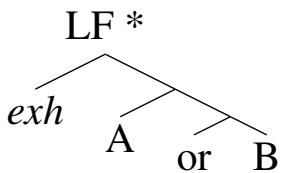

It is not the case that $\mathrm{LF}^{*}<\mathrm{LF} 3$. According to the definition of $<$ given below (6) above, $\mathrm{LF}^{*}$ would have to be derived from LF3 by substitution with sub-constituents and/or lexical items. Suppose we start with the following substitution, then:

$$
[[\operatorname{exh} \mathrm{A}] \text { or }[\text { not } \mathrm{A} \text { and } \underline{\mathrm{B}}]] \longrightarrow[[\operatorname{exh} \mathrm{A}] \text { or } \mathrm{B}]
$$

To get to $\mathrm{LF}^{*}$ from the right-hand structure would require an additional, illicit operation, namely, the movement of the locally adjoined exh to the matrix position:

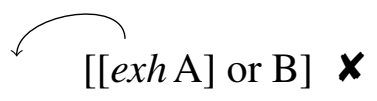

But according to the definition of $\lesssim$ (see (6) above), the movement operation does not preserve complexity and LF* $\nless$ LF3. It should be noted that the derivation in (18) does yield a structure that is strictly simpler than, LF3. But this structure is not semantically equivalent to LF3 and therefore not a competitor w.r.t. Efficiency. ${ }^{6}$

Efficiency thus correctly predicts that complex disjunctions like (3b) can be licensed. Crucially, though, this can only be achieved by (non-vacuous) embedded exhaustification. LF3 is thus the only licensed structure for (3b). ${ }^{7}$ The predicted interpretation is that of an an exclusive disjunction. This prediction is empirically

6 Suppose, as we have done for LF3, that the set $I E$ in the right-hand LF in (18) consists of just $B$. Then $\llbracket[[$ exh $\mathrm{A}]$ or $\mathrm{B}] \rrbracket=(A \wedge \neg B) \vee B=(A \vee B)$. LF3, however, expresses an exclusive disjunction. The same result (modulo additional implicatures for LF3) will be obtained when more alternatives $C, D, \ldots$ are in $I E$.

7 There is a fourth LF, which we haven't considered yet:

\section{(i) $\quad$ LF4}

$$
\begin{aligned}
& \text { [A or [not } \mathrm{A} \text { and }[\operatorname{exh} \mathrm{B}]]] \\
& =A \vee B
\end{aligned}
$$

By parity of reasoning let the alternative for exh be simply A, so that $I E=\{\mathrm{A}\}$. This will result in the inclusive disjunctive meaning shown above. LF4 is blocked because there is (at least) one simpler competitor that expresses this meaning: 
Redundancy and embedded exhaustification

correct, as the following data show (contra Mayr \& Romoli 2013):

(20) I'm not sure if Mary has two degrees, but...

a. \# She either studied math or she didn't and she studied physics

b. She either studied math or physics ${ }^{8}$

The contrast below makes the same point:

a. (Either) Mary studied math or she studied physics... perhaps even both.

b. (Either) Mary studied math or she didn't and she studied physics... \# perhaps even both.

One might argue that (21b) is ruled out by an independent constraint on anaphoric both. According to this argument, the antecedent of both needs to be a constituent of the form [A or B] which it then turns into the corresponding conjunctive meaning. As the only available antecedent of that form yields a contradiction when conjoined (i.e, she both studied math and didn't and studied physics), the continuation is ruled out by this independent factor. ${ }^{9}$

However, if this alternative explanation were on the right track, the following minimal variation should be just as degraded as (21b), which it isn't:

(Either) Mary studied math or she didn't and she studied physics... definitely not both.

Even though the possible antecedents for both are given in exactly the same configuration as in (21b), the continuation is felicitous. The only difference between (21b) and (22) is that the continuation in (21b) contradicts an exclusive disjunction meaning, while reinforcing it in (22). In sum, the contrast in (21), in conjunction with the observation in (22), is evidence in favor of the current proposal, specifically, the prediction that the only efficient LF for sentences like $A$ or not $A$ and $B$ yield an

(ii) $\quad \mathcal{C O M \mathcal { P }}([\mathrm{A}$ or $[$ not $\mathrm{A}$ and $[\operatorname{exh} \mathrm{B}]]])=\{[\mathrm{A}$ or $\mathrm{B}], \ldots\}$

LF3 remains the only efficient structure for the complex disjunction.

8 One might object that (21) involves a confound: The first sentence signals that the speaker is not informed about the alternative [math and physics]. This might be said to interfere with the availability of this alternative. If this were the case, (20b) would be expected to be acceptable a fortiori. The infelicity of (20a) would still follow from the proposed account: With the predicted LF3, embedded exh only needs physics as an alternative to make the whole structure express an exclusive meaning, inconsistent with the first sentence in (21).

9 Thanks to Irene Heim for pointing out this potential objection. 
exclusive disjunction meaning. ${ }^{10}$

SUMMARY Q-1 The goal of this paragraph was to find out why (3b) is licensed despite its seeming redundancy in view of the simple disjunction (3a). The principle of Efficiency was proposed as a formalization of the intuitive notion of redundancy. Applying it to the seemingly redundant structure $A$ or not $A$ and $B$ revealed that local exhaustification is at the heart of the felicity of this structure: Parsing it as LF3 yields an exclusive disjunctive meaning. Crucially, this LF, and this LF alone, is not blocked by another way of expressing (exclusive) disjunction, specifically, it is not blocked by exh [A or B]. This is an important finding, insofar as it points to the limits of structural competition with respect to Efficiency: A given LF competes only with a restricted set of structures - roughly, those which can be derived by substitution with subconstituents (and lexical items), but not those which involve syntactic movement in addition.

Addressing Q-2 We will now consider the contrast from (4) above, which was noted by Gajewski \& Sharvit (2012):
a. Mary studied math or physics or both.
b. \# Mary didn't study math or physics or both.

I have proposed an account of the Hurford disjunction in (23a) elsewhere, to which we will return briefly at the end of the paper. Here I want to concentrate on the infelicity of (23b). As we will see, Efficiency correctly predicts that there cannot be a non-redundant LF for this sentence. Following are the candidates and the competitors that block them:

$$
\begin{aligned}
& \text { LF1 } \\
& \text { not }[[\text { math or physics }] \text { or [math and physics }]] \\
& =\neg(\text { Mary studied } \text { math or physics }) \\
& \mathcal{C O} \mathcal{M P}(\mathrm{LF} 1)=\{\text { not }[\text { math or physics }], \ldots\} \\
& =\neg(\text { Mary studied math or physics })
\end{aligned}
$$

This competitor is derived via substitution with sub-constituents and is therefore simpler than LF1. As it is also semantically equivalent to LF1, LF1 is ruled out by Efficiency. As expected, vacuous exhaustification above the negation is likewise ruled out:

10 See Meyer (2015: 16) for a proposal on propositional anaphora under which both (21b) and (22) are predicted to have an available anaphor, derived from taking the constituent [Mary studied math or she didn't and she studied physics] and substituting the second disjunct by its subconstituent, to obtain [Mary studied math or she studied physics]. The fact remains that the observed contrast between (21b) and (22) could not be due to the anaphor both. 
Redundancy and embedded exhaustification

\section{LF2}

exh not [[math or physics] or [math and physics]]

$=\neg($ Mary studied math or physics $)$

The clause below exh denotes the negated disjunction, as seen in LF1. All alternatives for the exh-operator are therefore entailed by its argument: Mary didn't study math, Mary didn't study physics, Mary didn't study math and physics. Therefore, no alternative can be negated without contradiction, i.e., the set $I E$ is empty. The resulting meaning is thus the same as before, and so is the simpler competitor expressing this meaning:

$$
\begin{aligned}
& \mathcal{C O M P}(\mathrm{LF} 2)=\{\text { not }[\text { math or physics }], \ldots\} \\
& =\neg(\text { Mary studied math or physics })
\end{aligned}
$$

Exhaustification below negation, on the other hand, does have a semantic effect. An LF involving such embedded exhaustification might thus stand a chance of satisfying Efficiency:

\section{LF3}

not exh [[math or physics] or [math and physics]] $\mathcal{A L T}([[$ math or physics] or [math and physics $]])=$ $\{[$ math or physics], [math and physics], math, physics $\}$

$I E=\{[$ math and physics $]\}$

$$
=\neg[(\text { Mary studied math or physics }) \& \neg(\text { Mary studied math and physics })]
$$

The meaning expressed by this LF is more complex: Mary studied either both or none of the two. But is this LF efficient, i.e., is there a simpler equivalent competitor? It turns out that there is:

$$
\begin{aligned}
& \mathcal{C O M P}(\mathrm{LF} 3)=\{\text { not } \text { exh }[\text { math or physics }], \ldots\} \\
& =\neg[(\text { Mary studied math or physics }) \& \neg(\text { Mary studied math and physics })
\end{aligned}
$$

We will discuss structures like this competitor LF, which involve weakening effects of exhaustification, in the next section. For now, it suffices to note that the structure is predicted to block LF3. Let us lastly turn to the following possible LF:

\section{(30) LF4}

not [exh[math or physics] or [math and physics]]

$I E=\{[\text { math and physics }]\}^{11}$

$\neg[[($ math or physics) \& $\neg$ (math and physics) $]$ or (math and physics)]

11 This will be the set of excludable alternatives regardless of whether or not we include only [math and physics], i.e., the other disjunct, or the full Sauerland-set \{math, physics, math and physics . 


$$
=\neg(\text { Mary studied math or physics })
$$

Though exhaustification is not vacuous locally, the meaning of the LF as a whole is simply that of a negated disjunction. Again, there is a competitor with the same semantics:

$$
\begin{aligned}
& \mathcal{C O M P}(\mathrm{LF} 4)=\{\text { not }[\text { math or physics }], \ldots\} \\
& =\neg(\text { Mary studied math or physics })
\end{aligned}
$$

As a consequence, LF4 will be ruled out as well.

SUMMARY Q-2 In sum, the principle of Efficiency straightforwardly derives the infelicity of negated Hurford disjunctions like (23b). As we saw, all possible parses conflict with Efficiency - there is always a simpler structural competitor. Under the current proposal, this is the source of their infelicity.

\section{Extension: weakening implicatures}

Efficiency does not rule out cases where structural complexity incurs semantic weakening. In particular, the principle licenses exhaustification in the scope of downward-entailing operators like negation:

$$
\begin{aligned}
& \text { LF1 } \\
& \text { not } \operatorname{exh}[\mathrm{A} \text { or } \mathrm{B}] \\
& I E=\{[\mathrm{A} \text { and } \mathrm{B}]\}^{12} \\
& =\neg[(A \vee B) \wedge \neg(A \wedge B)] \\
& =\neg(A \vee B) \vee(A \wedge B)
\end{aligned}
$$

My claim here is that LFs like (32) are indeed attested, as Efficiency predicts. Specifically, I suggest that some cases of 'meta-linguistic negation' (Horn 1989) are an instance of these weakening embedded implicatures. ${ }^{13}$ Consider (33):

12 The specific make-up of $I E$ is based on the assumption that $\mathcal{A} L T$ is the Sauerland-set. If there is another alternative for the whole disjunction, e.g., $C$, the meaning will be different. Most likely, this meaning can only be expressed with only, cf. John didn't \#\{only\} ask Mary or Sue... He asked Bill as well, and not with the LF above. As we will see below, these assumptions about (32) are consistent with facts about focus-marking: For the weakening implicature to arise, the disjunction needs to receive pitch accent, which I will argue is part of a complex topic marking. I assume this indicates F-marking on or. Following Fox \& Katzir (2011); Fox \& Spector (2013), only the F-marked constituents (here: the terminal node or) is targeted by substitution (here: with and), yielding the assumed alternative set and $I E\{[\mathrm{~A}$ and $\mathrm{B}]\}$.

13 Horn himself pointed out that standard negation could cover his 'meta-liguistic' uses under the —at the time unthinkable - assumption that scalar implicatures arise during semantic computation: 'Such examples cannot be collapsed under Karttunen \& Peter's [contradiction negation] approach with- 
Redundancy and embedded exhaustification

Mary didn't study math OR physics... she studied both!

In what follows I will sketch an account of (33) in terms of embedded exhaustification, i.e., an LF like (32). I propose a compositional account of how the characteristic intonational pattern associated with (33) disambiguates in favor of the LF in (32) (this is question Q-1). I will then discuss open issues pertaining to the the role of the continuation ... both! (question Q-2).

Addressing Q-1 It is well-known that there is a characteristic intonational contour associated with (33). A closer look reveals that phonological focus, i.e., pitch accent on or, is insufficient to disambiguate in favor of the weakening implicature (contra Fox \& Spector 2013):
A: Mary DIDN' $\mathrm{T}_{\mathrm{H}^{*}}$ buy the Porsche $\mathrm{OR}_{\mathrm{H}^{*}}$ the Mercedes $\mathrm{LL}$.
B: So she didn't buy any car?
C: \# So she bought two cars?

The final fall in (34) seems to re-enforce the strong reading brought about by LFs like not [A or B]. The weakening implicature, by contrast, requires a final rise in addition to the pitch accent. Interestingly, it seems to be the pattern associated with contrastive topics (s. Jackendoff 1972; Büring 2003): ${ }^{14}$
A: Mary DIDN' $\mathrm{T}_{\mathrm{H}^{*}}$ buy the Porsche $\mathrm{OR}_{\mathrm{L}+\mathrm{H}^{*}}$ the Mercedes $\mathrm{LH}_{\mathrm{L}}$.
B: $\checkmark$ So she bought two cars?
C: \# So she didn't buy any car? ${ }^{15}$

But just what is the role of the CT-contour $\mathrm{L}+\mathrm{H}^{*} \mathrm{LH} \%$ in cases like (33)? As stated earlier, my suggestion is that the CT-contour disambiguates in favor of an LF as in (32). To derive this formally, I follow Büring (2003) in assuming that the contour comes with the following inference:

At least one question in $\llbracket \phi \rrbracket^{\mathrm{CT}}$ is left open after asserting $\phi$

out incorporating conversational implicata (...) into the logical form of these sentences [emphasis mine]. But conversational implicata by definition are not part of logical form.' (Horn 1989:370)

$14 \mathrm{It}$ is often assumed that the pitch accent on contrastive phrases involves a steeper rise, i.e., $\mathrm{L}+\mathrm{H}^{*}$, which is why I marked it as such here. See e.g., Hirschberg \& Pierrehumbert (1986); Watson, Tanenhaus \& Gunlogson (2008). Though Horn discussed the similarity to Jackendoff's 'B-accent' he did not put forward a formal proposal about the exact semantic contribution of this contour in utterances like (33).

15 The infelicity of this follow-up question is an instance of the observation discussed below, namely, that ...neither continuations are impossible under CT-intonation. Given this fact, the oddness of $C$ 's reaction indicates that under the given intonation, only the weakening not exh [A or B] parse is available, but not the strong parse not [A or B]. 
$\llbracket \phi \rrbracket^{\mathrm{CT}}$ is the contrastive topic semantic value of $\phi$ and can be thought of as a set of question meanings. Looking at the LF in (32) involving the weakening exh-operator, we can now ask what CT-inference this LF would give rise to. As can be seen from (36), this will depend on its CT-value, which is given below (see Büring 2003 for details): ${ }^{16}$

$$
\begin{aligned}
& \llbracket \operatorname{not}_{\mathrm{F}} \operatorname{exh}\left[\mathrm{A} \text { or }_{\mathrm{CT}} \mathrm{B}\right] \rrbracket^{\mathrm{CT}}= \\
& \{A \text { or B but not both?, } A \text { and } B ?\}
\end{aligned}
$$

According to (36), the CT-inference associated with this LF is that either the question whether $A$ or $B$ but not both, or the question whether $A$ and $B$ are left open after the assertion, i.e., by the standard semantic value of this LF, which is given below:

$$
\begin{aligned}
& \llbracket \operatorname{not}_{\mathrm{F}} \operatorname{exh}\left[\mathrm{A} \text { or }_{\mathrm{CT}} \mathrm{B}\right] \rrbracket \\
& =\neg[(A \vee B) \wedge \neg(A \wedge B)] \\
& =\neg(A \vee B) \vee(A \wedge B)
\end{aligned}
$$

(38) leaves open the last question in the CT-value above, namely, whether $A$ and $B$. This is exactly the open issue that the continuation subsequently resolves.

What happens if a sentence involving the same focus- and CT-marking is parsed without embedded exh? The CT-inference is predicted to be different, as is the standard semantic value:

$$
\begin{aligned}
& \text { LF2 } \\
& \operatorname{not}_{\mathrm{F}}\left[\mathrm{A} \text { or }_{\mathrm{CT}} \mathrm{B}\right] \\
& \llbracket \mathrm{LF} 2 \rrbracket=\neg(A \vee B) \\
& \llbracket \mathrm{LF} 2 \rrbracket^{\mathrm{CT}}=\{A \text { or } B ?, \text { A and } B ?\}
\end{aligned}
$$

Importantly, the CT inference brought about by the intonational contour is false under LF2. This is because the basic semantic meaning is very strong: $\neg(A \vee B)$. As such, it answers both questions in the CT-value, i.e., whether $A$ or $B$ (no!) and whether $A$ and $B$ (again no!).

The foregoing discussion is summarized in the diagram below:

16 I assume that the negation is focused, as indicated by its pitch accent. Again I use sloppy notation in that the italicized questions are short for their semantic denotations. 
Redundancy and embedded exhaustification
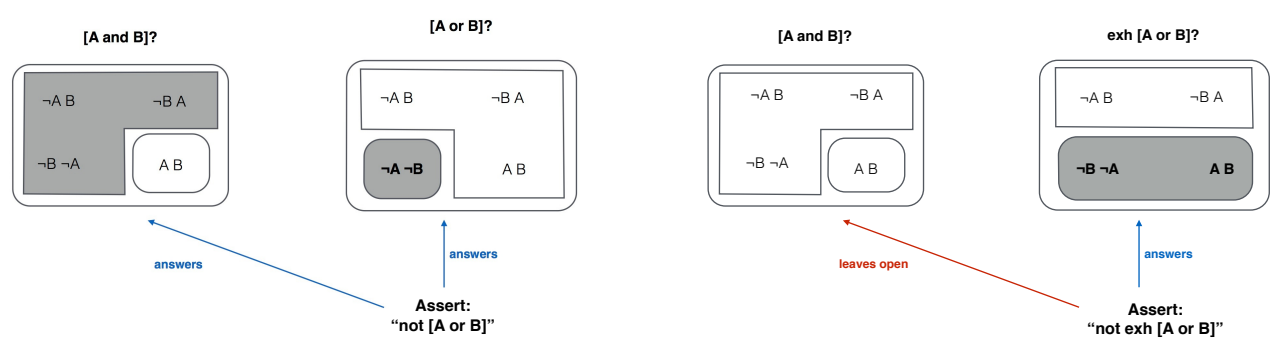

Figure 1 Does the assertion leave open a question in the CT-value? Difference between LF with embedded exh and simple LF.

Independent evidence that the intonational pattern comes with an inference along these lines comes from the following contrast (see Büring 2003 for detailed discussion):

$$
\begin{aligned}
& \text { Mary solved } \mathrm{SOME}_{\mathrm{L}+\mathrm{H}^{*}} \text { of the } \mathrm{p} \text {-sets } \mathrm{LH} \% \\
& \llbracket \rrbracket^{\mathrm{CT}}=\{\text { did Mary solve some of the p-sets?, did Mary solve all of the p-sets? }\}
\end{aligned}
$$

\# Mary solved $\mathrm{ALL}_{\mathrm{L}+\mathrm{H}^{*}}$ of the p-sets $\mathrm{LH} \%$.

$\llbracket \rrbracket^{\mathrm{CT}}=\{$ did Mary solve some of the p-sets?, did Mary solve all of the p-sets?\}

In (40), the underlined question in the CT-value is still left open by the assertion, and the sentence is felicitous. But in (41), no question is left open, and the sentence sounds odd under the given contour. ${ }^{17}$

We are now in a position to formulate a hypothesis about Q-1: what is the role of the intonational pattern? As suggested by Büring (1997), CT-inferences may act as 'a filter on LFs' in that those structures which falsify the corresponding inference are blocked. As seen by the oddness of (41), if no other parse is available to circumvent a conflict with the CT-inference, a sentence may become infelicitous. In the case of our initial sentence $\operatorname{not}_{F} A \operatorname{or}_{C T} B$, only the LF containing embedded exhaustification satisfies the relevant CT-inference, whereas the LF without the embedded implicature does not. Thus, assuming a syntactic operator $e x h$, the role of intonation in 'metalinguistic' negation like (33) can be unified with the scope-reversal cases discussed by Jackendoff and Büring: via its semantic contribution, the intonational contour disambiguates between different LFs.

17 As can be seen from the CT-values, I assume that these sentences include verum focus which is not realized phonetically. Note also that the LF exh [Mary solved SOME of the p-sets] would conflict with the CT-inference as well, though re-interpretation as in (40) prevents the sentence from sounding odd. Lastly, a different intonational contour, with the pitch accent realized on $p$-sets, can make (41) acceptable. This is predicted given principles of focus projection (e.g., Selkirk 1995). 
So far, we predict that when the contour is used, the only available LF will contain an embedded, weakening implicature. But the implication seems to hold in the other direction as well: Under the 'meta-linguistic' use, the only available intonation is the CT-contour, at least for the speakers I have consulted so far (s. (34)). Assume that these intuitions can be substantiated by more systematic empirical investigation. Following Roberts (1996), the CT-inference in (36) could be modeled as a presupposition, making its use subject to Heim's Maximize Presupposition (Heim 1991). ${ }^{18}$

Summarizing thus far, (42) has to be parsed as LF1 given its intonation:

$$
\begin{aligned}
& \text { Mary didn't study math OR physics }{ }_{\mathrm{LH} \%} \ldots \\
& \text { LF1: not } \operatorname{exh}[\mathrm{A} \text { or B] } \\
& =\neg(A \vee B) \vee(A \wedge B)
\end{aligned}
$$

Addressing Q-2 Semantically, LF1 is consistent with Mary having studied both subjects, and none of the two. ${ }^{19}$ The role of the continuation seems to be to fully resolve this issue. But, as illustrated below, whereas the ... both continuation is possible, perhaps even obligatory for (42), the equally consistent continuation in (43b) is highly marked:

Mary DIDN'T study math OR physics $L$ H\%...

a. she studied both.

b. ?? she didn't study anything/she studied neither.

At first it seems that this asymmetry follows from independent constraints on focus placement in contrastive negation. Specifically, if a statement $v$ is negated and contrasted with an alternative statement $\alpha$, focus-placement in $v$ is subject to constraints that are partly determined by $\alpha$ (and vice versa). This is illustrated below (e.g., Rooth 1992):

18 Note incidentally that this offers the perspective of a competition between the falling contour H*LL\% as in (34) and the rising $\mathrm{L}+\mathrm{H}^{*} \mathrm{LH} \%$ contour: The former would give rise to the anti-presupposition that the presupposition of the CT-inference is not met (Percus 2006; Sauerland 2008), i.e., that there are no open questions in the CT-value. For a sentence like John ate SOME of the cookies (with verum focus), the falling contour would then anti-presuppose that one question in \{did John eat some of the cookies?, did John eat all of the cookies?\} is left open. This, in turn, can only be satisfied if exh is adjoined. Reversely, with raising intonation a question in this value would have to be left open, which is only possible without exh. Further research is needed to establish whether the predicted correlation between falling vs. raising intonation and presence vs. absence of implicatures can be substantiated.

19 In an unpublished manuscript, Fox \& Spector (2013) argue for an LF of (33) under which the sentence would have a conjunctive meaning: Mary studied math and physics. Under this proposal, it is unclear why the continuation... she studied both! is even allowed, and even more mysterious why it would be needed. 
Redundancy and embedded exhaustification

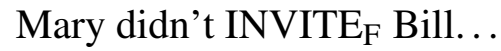

a. \# she invited FRED .

b. $\checkmark \checkmark$ she SUMMONED $\mathrm{F}$ him.

Uncontroversially, I assume that the pitch accent on OR reflects abstract F-marking on the connective, ${ }^{20}$ which in turn means that the alternatives which (43) can be contrasted with are just the singleton set in (45a):

$$
\begin{array}{ll}
\text { a. } & \mathcal{A L T}(\mathrm{A} \text { or } \mathrm{F} \mathrm{B})=\{\mathrm{A} \text { and } \mathrm{B}\} \\
\text { b. } & \mathcal{A L T}\left([\mathrm{A} \text { or } \mathrm{B}]_{\mathrm{F}}\right)=\{\mathrm{C}, \mathrm{D}, \ldots\}
\end{array}
$$

The prediction is that not only ... neither, but also other continuations that do not obey the relevant parallelism constraint should be ruled out. This prediction is borne out:
a. Mary DIDN'T study math OR physics LH\% ...\# she studied CHEM- ISTRY
b. Mary DIDN'T study [math or PHYSICS $]_{\text {LH\% }} \ldots \checkmark$ she studied CHEM- ISTRY

Though this might be an independent explanation as to why the neither continuation in $(43 \mathrm{~b})$ is ruled out, it doesn't carry over to the following case, where it predicts (47b) to be acceptable:

Mary DIDN'T read SOME of the books $\mathrm{LH}_{\%} \ldots$

a. she read all of them.

b. ?? she read none of them.

As far as I have been able to check, (47b) is still marked when compared to (47a). I cannot provide an answer to this puzzle here, except to note that in addition to the above constraint on focus placement, another factor in the asymmetry between the two semantically possible resolutions might be the overall QUD that's being addressed, for instance, What did Mary study? or How many of the books did Mary read?. It has been argued that wh-questions carry an existential presupposition (Karttunen \& Peters 1979; Comorovski 1996). An analogous requirement on the common ground might hold for $Q U D s$, i.e., the current topic of conversation. In a context where what Mary studied is being discussed, resolving a sentence that is semantically consistent with Mary having studied neither or both of the two relevant subjects in favor of the former possibility would negate the appropriateness of the current conversational topic. Only the ... both resolution is in line with the relevant

20 See Krifka (2007) for the relation between CTs and F-marking. 
requirement on the common ground that there is something that Mary studied. ${ }^{21}$

In closing this section I would like to briefly illustrate the suggestion with another DE-operator, exploring its potential to extend to weakening implicatures beyond the case of negation.

(48) EVERYbody F $_{\text {who failed the oral }} \mathrm{OR}_{\mathrm{CT}}$ the written exam passed... but nobody who failed both did.

Consider the LF with exh embedded in the restrictor of the universal quantifier:

\section{LF1}

everybody who exh [failed the oral or the written exam] passed

$\llbracket \mathrm{LF} 1 \rrbracket=\forall x:[O(x) \vee W(x) \wedge \neg(O(x) \wedge W(x))] \rightarrow P(x)$

$\llbracket \mathrm{LF} 1 \rrbracket^{\mathrm{CT}}=\{$ how many who did $O$ or $W$ but not both passed?, how many who did $O$ and $W$ passed?\}

The ordinary semantic value of LF1 leaves open the last question, i.e., how many of those who failed both the oral and the written exam passed. ${ }^{22}$ By contrast, if there is no embedded exh the CT-inference will be false:

\section{LF2}

everybody who [failed the oral or the written exam] passed

$\llbracket \mathrm{LF} 2 \rrbracket=\forall x: O(x) \vee W(x) \rightarrow P(x)$

$\llbracket \mathrm{LF} 2 \rrbracket^{\mathrm{CT}}=\{$ how many who did $O$ or $W$ passed?, how many who did $O$ and W passed?\}

The meaning of LF2 answers both questions. Therefore, LF1 containing weakening embedded exh satisfies the CT-inference, but LF2 does not. The case is thus parallel to the previously discussed example involving negation.

SUMMARY: WEAKENING IMPLICATURES In sum, the LFs involving embedded exh below negation or in the restrictor of a universal quantifier satisfy the inference associated with the CT-contour, but those LFs without embedded exhaustification do

21 It should be kept in mind that this line of explanation may lead to the same issues raised in the previous footnote with respect to Fox \& Spector's proposal: Together with this requirement ('presupposition') of the $Q U D$, the sentence would come to mean that Mary studied math and physics. The redundancy (!) of the ... both continuation would again be puzzling. Differences in the level of meaning might play a role here, namely, whether the conjunctive meaning corresponds to the semantic denotation (as in Fox \& Spector's proposal) or whether it is derived from context in conjunction with the semantics.

22 The additional scalar implicature that $\neg$ (everybody who failed the oral and the written exam passed the class) would not answer the question either (it could be no students or some). Under the grammatical view taken here, this implicature would be derived by an additional exh in matrix position. As a consequence, the intonational contour does not rule out such an LF, as long as there is also an embedded $e x h$. 
not. Why is the CT-contour required for weakening implicatures as in LF2? This concerns the status of the CT-inference: It might be a presupposition, positioning the obligatory intonational marking of weakening implicatures within the scope of Heim's Maximize Presupposition principle (Heim 1991). This, however, conflicts with evidence suggesting that CT-intonation is not obligatory when it is used to signal discourse structure (s. Meyer, Fedorenko \& Gibson 2012). Further research is needed to establish (i) whether intonational marking of weakening implicatures is in fact obligatory and (ii) at what level of meaning the CT-inference is located.

\section{Outlook: back to Hurford}

In section 2 we saw how the infelicity of (51b) follows from Efficiency: For all possible LFs, there exists a simpler equivalent competitor which blocks it. By the same logic, we expect to find at least one LF which satisfies Efficiency for (51a), as the sentence is felicitous:

a. Mary studied math or physics or both.

b. \# Mary didn't study math or physics or both.

However, it turns out that all possible LFs for (51a) are blocked by a simpler competitor. To briefly illustrate this, consider two possible LFs for (51a): ${ }^{23}$

$$
\begin{array}{ll}
\text { a. } & \mathbf{L F 1}[[\mathrm{A} \text { or } \mathrm{B}] \text { or }[\mathrm{A} \text { and } \mathrm{B}]] \\
\text { b. } & \mathbf{L F 2}[\operatorname{exh}[\mathrm{A} \text { or } \mathrm{B}] \text { or }[\mathrm{A} \text { and } \mathrm{B}]]
\end{array}
$$

The meaning of these two LFs is $A \vee B$. Both are blocked by the same competitor:

$$
\mathcal{C O} \mathcal{M P}(\mathrm{LF} 1, \mathrm{LF} 2)=\{[\mathrm{A} \text { or B }], \ldots\}
$$

Likewise, LF3 $=\operatorname{exh}[[\mathrm{A}$ or $\mathrm{B}]$ or $[\mathrm{A}$ and $\mathrm{B}]]$ will have the meaning of an exclusive disjunction, but will be blocked by the competitor exh[A or B].

Therefore, whatever the correct LF will turn out to be (and its felicity tells us that there must be one), the prediction is that (51a) will not have a standard inclusive or exclusive disjunction meaning. In previous work I argued that this is in fact a correct prediction, because the meaning of (51a) (semantically) conveys true ignorance ${ }^{24}$ with respect to the corresponding conjunction and thereby differs from simpler

23 As stated earlier, I do not want to commit to the claim that both is represented as a full conjunctive phrase at LF; for present purposes it suffices to make the innocuous assumption that its meaning is equivalent to [A and $\mathrm{B}]$.

24 By true ignorance I mean uncertainty that is inconsistent with certainty about the corresponding negation. Simply put: $\neg K(\phi) \wedge \neg K(\neg \phi)$, as opposed to $\neg K(\phi)$, i.e., uncertainty about $\phi$, which is consistent with $K(\neg \phi)$. 
disjunctions (see Meyer 2013: ch. 2.3 for details).

We now have two observations: First, (51a) is felicitous despite blocking competitors for both the inclusive and the exclusive disjunction meaning. Secondly, (51a) has a meaning component which distinguishes it from standard (inclusive or exclusive) disjunction: true ignorance about the conjunction. Here I would like to offer the following perspective on these observations. I suggest that the ignorance inference about the conjunction arises as a rescue strategy to make (51a) efficient after all. In other words, the only way (51a) can avoid blocking by a simpler competitor is to somehow acquire the observed true ignorance meaning. My claim as to how this comes about is this:

\section{Rescuing by $\mathrm{K}$}

An operator K expressing doxastic necessity relative to the speaker can be inserted at matrix position 25

Simply adjoining a matrix K won't result in an efficient LF for (51a). ${ }^{26}$ Rather, Efficiency predicts exactly one possible LF for the sentence, which involves embedded exhaustification:

$$
\begin{aligned}
& \text { Efficient LF for (51a) } \\
& \text { exh } K[\operatorname{exh}[\mathrm{A} \text { or B] or [A and B]] } \\
& =K(A \vee B) \wedge \neg K(A \wedge B) \wedge \neg K \neg(A \wedge B)
\end{aligned}
$$

As shown, the meaning of this LF indeed entails true ignorance with respect to $A$ and $B$. But if $K$ can be parsed at matrix position to rescue otherwise inefficient structures, can we still derive the infelicity of the structure we started out with, i.e., \# not [A or $B$ or both]? It turns out that $K$ will not be able to rescue this sentence: All possible configurations of $K$ and $e x h$ have a simpler equivalent competitor. ${ }^{27}$

Here I cannot develop the envisaged rescuing account for (51a) in more detail. A crucial ingredient will be independent arguments for the existence of the proposed K-rescue strategy. Indeed, a doxastic universal operator has been assumed precisely

25 I assume that exh may outscope K.

26 For instance, just adjoining it without exhaustifying will yield LF4 $=\mathrm{K}[[\mathrm{A}$ or B] or [A and B]]. As is obvious, this LF will be blocked by the simpler K[A or B].

27 This is illustrated for the least obvious candidate LF below:

(i) LF1 exh $\mathrm{K}$ not $\operatorname{exh}[$ [A or B] or [A and B]]

$$
=K[\neg(A \vee B) \vee(A \wedge B)] \wedge \neg K(\neg(A \vee B)) \wedge \neg K(A \wedge B) \wedge \neg K(A \vee B)
$$

$$
\begin{aligned}
& \mathcal{C O} \mathcal{M P}(L F 1)=\{\text { exh } \mathrm{K} \text { not } \operatorname{exh}[\mathrm{A} \text { or } \mathrm{B}]\} \\
& =K[\neg(A \vee B) \vee(A \wedge B)] \wedge \neg K(\neg(A \vee B)) \wedge \neg K(A \wedge B) \wedge \neg K(A \vee B)
\end{aligned}
$$


Redundancy and embedded exhaustification

in this role in accounts of such diverse phenomena as Free Choice disjunction, modal indefinites and modal auxiliaries, among others (e.g., Kratzer \& Shimoyama 2002; Alonso-Ovalle 2006; Hacquard 2010).

\section{References}

Alonso-Ovalle, Luis. 2006. Disjunction in Alternative Semantics: UMass Amherst $\mathrm{PhD}$ dissertation.

Büring, Daniel. 1997. The great scope inversion conspiracy. Linguistics and Philosophy 20(2). 175-194. doi:10.3765/salt.v5i0.3116.

Büring, Daniel. 2003. On D-Trees, Beans, and B-Accents. Linguistics and Philosophy 26. 511-545.

Comorovski, Ileana. 1996. Interrogative Phrases and the Syntax-Semantics Interface. Amsterdam: Kluwer.

Fox, Danny. 2007. Free Choice and the theory of scalar implicatures. In Uli Sauerland \& Penka Stateva (eds.), Presupposition and Implicature in Compositional Semantics, 71-120. New York: Palgrave Macmillan.

Fox, Danny \& Roni Katzir. 2011. On the characterization of alternatives. Natural Language Semantics 19(1). 87-107. doi:10.1007/s11050-010-9065-3.

Fox, Danny \& Benjamin Spector. 2013. Economy and embedded exhaustification. Ms., MIT and ENS.

Gajewski, Jon \& Yael Sharvit. 2012. In defense of the grammatical approach to local implicatures. Natural Language Semantics 20. 31-57. doi:10.1007/s11050-0119074-x.

Hacquard, Valentine. 2010. On the event relativity of modal auxiliaries. Natural Language Semantics 18. 79-114. doi:10.1007/s11050-010-9056-4.

Heim, Irene. 1991. Artikel und Definitheit. In A. von Stechow \& D. Wunderlich (eds.), Semantics: An International Handbook of Contemporary Research, 487535. Berlin: de Gruyter.

Hirschberg, Julia \& Janet Pierrehumbert. 1986. The intonational structuring of discourse. In 24th Annual Meeting of the Association for Computational Linguistics, 136-144. New York.

Horn, Laurence. 1989. A Natural History of Negation. Chicago, IL: University of Chicago Press.

Jackendoff, Ray. 1972. Semantic Interpretation in Generative Grammar. Cambridge, MA: MIT Press.

Karttunen, Lauri \& Stanley Peters. 1979. Conventional implicature. In Choon-Kyu Oh \& David A. Dinneen (eds.), Syntax and Semantics 11: Presupposition, 1-55. New York: Academic Press. 
Katzir, Roni. 2007. Structurally-defined alternatives. Linguistics and Philosophy 30(6). 669-690. doi:10.1007/s10988-008-9029-y.

Kratzer, Angelika \& Junko Shimoyama. 2002. Indeterminate pronouns: The view from Japanese. In Yukio Otsu (ed.), Third Tokyo Conference on Psycholinguistics, 1-25. Tokyo: Hituzi Syobo.

Krifka, Manfred. 2007. Basic notions of information structure. In Caroline Féry \& Manfred Krifka (eds.), Interdisciplinary Studies on Information Structure 6, 13-55. Universitätsverlag Potsdam.

Mayr, Clemens \& Jacopo Romoli. 2013. Redundancy and the notion of local context. Ms., ZAS and University of Ulster.

Meyer, Marie-Christine. 2013. Ignorance and Grammar: MIT PhD dissertation.

Meyer, Marie-Christine. 2014. Deriving Hurford's constraint. In T. Snider, S. D'Antonio \& M. Weigand (eds.), Semantics and Linguistic Theory (SALT) XXIV, 577-596. doi:10.3765/salt.v24i0.2518.

Meyer, Marie-Christine. 2015. Generalized Free Choice and missing alternatives. Journal of Semantics advanced access November 4, 2015. doi:10.1093/jos/ffv010.

Meyer, Marie-Christine, Evelina Fedorenko \& Edward Gibson. 2012. Contrastive Topic intonation: An empirical (re)-evaluation. Unpublished Ms., MIT.

Percus, Orin. 2006. Antipresuppositions. In A. Ueyama (ed.), Theoretical and Empirical Studies of Reference and Anaphora: Toward the Establishment of Generative Grammar as Empirical Science. Grant-in-Aid for Scientific Research (B), Project 15320052, 52-73. Tokyo: Japan Society for the Promotion of Science.

Roberts, Craige. 1996. Information structure in discourse: Towards and integrated formal theory of pragmatics. OSU Working Papers in Linguistics 49. 91-136.

Rooth, Mats. 1992. A theory of focus interpretation. Natural Language Semantics 1. 75-116. doi:10.1007/bf02342617.

Sauerland, Uli. 2008. Implicated presuppositions. In A. Steube (ed.), The Discourse Potential of Underspecified Structures, 581-600. Berlin: Mouton de Gruyter.

Sauerland, Uli. 2011. The computation of scalar implicatures: Pragmatic, lexical or grammatical? Language and Linguistics Compass 10. 1-14. doi:10.1002/lnc3.321.

Selkirk, Elisabeth. 1995. Sentence prosody: Intonation, stress, and phrasing. In John A. Goldsmith (ed.), The Handbook of Phonological Theory, 550-569. Blackwell.

Trinh, Tue \& Andreas Haida. 2015. Constraining the derivation of alternatives. Natural Language Semantics 23. 249-270. doi:10.1007/s11050-015-9115-y.

Watson, Duane, Michael Tanenhaus \& Christine Gunlogson. 2008. Interpreting pitch accents in online comprehension: $\mathrm{H}^{*}$ vs. $\mathrm{L}+\mathrm{H}^{*}$. Cognitive Science 32(7). 
Redundancy and embedded exhaustification

1232-244. doi:10.1080/03640210802138755.

\author{
Marie-Christine Meyer \\ Language, Logic and Cognition Center \\ The Hebrew University of Jerusalem \\ Mount Scopus \\ 91905 Jerusalem \\ macrst@alum.mit.edu
}

